\title{
A Proof of Concept: Grizzly, the LWRS Program Materials Aging and Degradation Pathway Main Simulation Tool
}

Ben Spencer Jeremey Busby Richard Martineau Brian Wirth

October 2012

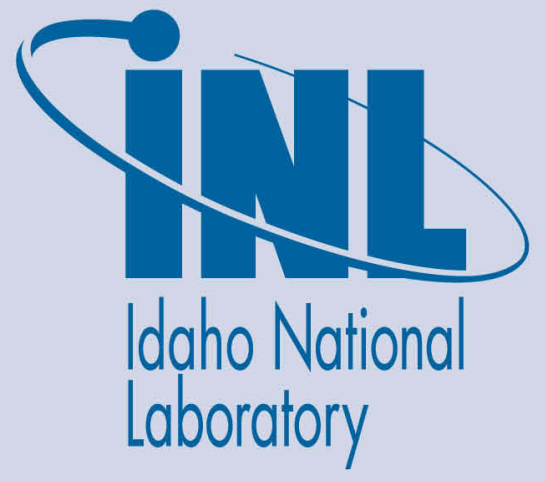

The INL is a U.S. Department of Energy National Laboratory operated by Battelle Energy Alliance 
INL/EXT-12-27559

\title{
A Proof of Concept: Grizzly, the LWRS Program Materials Aging and Degradation Pathway Main Simulation Tool
}

\author{
Ben Spencer \\ Jeremey Busby ${ }^{1}$ \\ Richard Martineau \\ Brian Wirth ${ }^{2}$ \\ ${ }^{1}$ ORNL \\ ${ }^{2}$ UTK
}

October 2012

\begin{abstract}
Idaho National Laboratory
Idaho Falls, Idaho 83415
\end{abstract}

http://www.inl.gov

Prepared for the

U.S. Department of Energy

Office of Nuclear Energy

Under DOE Idaho Operations Office

Contract DE-AC07-05ID14517 


\title{
A Proof of Concept: Grizzly, the LWRS Program Materials Aging and Degradation Pathway Main Simulation Tool
}

\author{
By \\ Ben Spencer (INL) \\ Jeremey Busby (ORNL) \\ Richard Martineau (INL) \\ Brian Wirth (UTK)
}

\section{Introduction}

Nuclear power currently provides a significant fraction of the United States' noncarbon emitting power generation. In future years, nuclear power must continue to generate a significant portion of the nation's electricity to meet the growing electricity demand, clean energy goals, and ensure energy independence. New reactors will be an essential part of the expansion of nuclear power. However, given limits on new builds imposed by economics and industrial capacity, the extended service of the existing fleet will also be required.

Ensuring public safety and environmental protection is a prerequisite to all nuclear power plant operating and licensing decisions at all stages of reactor life. This includes the original license period of 40 years, the first license extension to 60 years, and certainly for any consideration of life beyond 60 years. For extended operating periods, it must be shown that adequate aging management programs are present or planned and that appropriate safety margins exist throughout the subsequent license renewal periods. Materials degradation can impact reactor reliability, availability, and potentially, safe operation. Components within a reactor must tolerate the harsh environment of high temperature water, stress, vibration, and/or an intense neutron field. Degradation of materials in this environment can lead to reduced performance, and in some cases, sudden failure. Clearly, understanding materials degradation and accounting for the effects of a reactor environment in operating and regulatory limits is essential.

The Light Water Reactor Sustainability (LWRS) Program is designed to support the long-term operation (LTO) of existing domestic nuclear power generation with targeted collaborative research programs into areas beyond current short-term optimization opportunities [1]. Within the LWRS program, four pathways have been initiated to perform research essential to informing relicensing decisions [1]. The Materials Aging and Degradation Pathway is designed to help develop the scientific basis for understanding and predicting long-term environmental degradation behavior of materials in nuclear power plants and to provide data and methods to 
assess performance of systems, structures, and components essential to safe and sustained operation.

\section{Main Vulnerabilities Identified by the LWRS Program}

Reactor pressure vessels: Reactor pressure vessels are critical safety-related components in nuclear power plants. Repairing or replacing the pressure vessel is not practical, yet its mechanical integrity must be conservatively demonstrated for up to 80 years of operation. During operation, neutrons from the nuclear core impinge on the vessel wall, reducing its strength and ductility and diminishing its ability to withstand flaws that might be present, such as those that may have been introduced during fabrication. Research is ongoing in LWRS in the area of RPV embrittlement for extended service, building on decades of research designed to establish safe operating limits for service up to 60 years. As a result, there is a considerable experimental database and multiple models and tools for predicting performance and establishing safety limits under a variety of normal and off-normal operating conditions.

Concrete structures: Concrete structures are a key component within a nuclear power plant, providing support to all other components and providing a final containment boundary. As a result, the degradation of concrete structures may be a key safety concern during extended service if the concrete structures degrade. Research into concrete structure degradation has been initiated within LWRS and other programs around the world. However, concrete structures may not be useful for a case study. While of high value from a safety perspective, there is very little existing surveillance data (which is not specifically required by regulators) making it difficult to establish adequate modeling tools. Further, there are no existing models of concrete performance, further limiting the utility of this area for a case study.

Stress-corrosion cracking: Within a nuclear reactor system, a number of different corrosion mechanisms are at work. When combined with stress, stress-corrosion cracking (SCC) can and does occur in numerous alloys and locations within the reactor. Extended service will increase exposure to coolant, stress, and neutrons further increasing susceptibility to SCC. SCC is an area of active research in many organizations worldwide, including the LWRS program. SCC of different materials and components also receives considerable regulatory interest. However, despite over thirty years of research, the fundamental mechanisms of SCC are relatively unknown. Further, there is no existing model or tool for the prediction of SCC, making an immediate impact in a case study difficult. 


\section{Grizzly, the LWRS Program Materials Aging and Degradation Pathway Main Simulation Tool}

Grizzly is a MOOSE-based tool for simulating component ageing and damage evolution events for LWRS specific applications. The Multi-physics Object Oriented Simulation Environment (MOOSE) is the INL development and runtime environment for the solution of multi-physics systems that involve multiple physical models or multiple simultaneous physical phenomena. The systems are generally represented (modeled) as a system of fully coupled nonlinear partial differential equation systems (an example of a multi-physics system is the thermal feedback effect upon neutronics cross-sections where the cross-sections are a function of the heat transfer). Inside MOOSE, the Jacobian-Free Newton Krylov (JFNK) method is implemented as a parallel nonlinear solver that naturally supports effective coupling between physics equation systems (or Kernels). The physics Kernels are designed to contribute to the nonlinear residual, which is then minimized inside of MOOSE. MOOSE provides a comprehensive set of finite element support capabilities (libMesh) and provides for mesh adaptation and parallel execution. The framework heavily leverages software libraries from DOE SC and NNSA, such as the nonlinear solver capabilities in either the Portable, Extensible Toolkit for Scientific Computation (PETSc) project or the Trilinos project.

Specifically, Grizzly will provide a simulation capability for:

- Reactor Metals (embrittlement, fatigue, corrosion, etc.), such as Reactor Pressure Vessel (RPV) and core internals

- Weldment integrity

- Concrete integrity

subjected to a neutron flux, corrosive environment, and high temperatures and pressures. As with other applications utilizing the ever-growing library of MOOSE physics Kernels, Grizzly will heavily leverage the thermo-mechanics physics found in the BISON fuels performance application as a starting point.

\section{RPV Case Study}

All three areas of research were initially discussed. However, the area of RPV steels and embrittlement was quickly determined to be the most viable and desirable for collaboration, being the only topic satisfying all the criteria listed above. This active area of research has been the recent subject of research for both the current and extended operating periods.

One specific area of RPV research has been specifically suggested as a case study. Pressure vessel embrittlement is of particular concern in PWRs due to pressurized thermal shock (PTS). PTS can occur under some accident scenarios where cold water is introduced into a reactor pressure vessel that subsequently repressurizes. 
The cold water causes the vessel to cool rapidly, resulting in large thermal stresses, which could initiate cracks that could propagate during repressurization in the embrittled vessel material, possibly to the point of breaching the vessel wall. 22Although such a failure has never happened, the U.S. Nuclear Regulatory Commission (NRC) issued a rule in the mid-1980s (10 CFR part 50.61, the "PTS Rule") that limits the amount of embrittlement before additional evaluations or corrective actions are required. Operators of older nuclear plants found it extremely difficult to adequately demonstrate reactor pressure vessel integrity using the 1970s-vintage analytical assumptions embodied in the original PTS rule. One plant, Yankee Rowe, shut down pre-maturely in 1992 due, in part, to the high cost and difficulty of demonstrating pressure vessel integrity under postulated PTS conditions. Several other plants, including Palisades, Beaver Valley and Kewaunee, also faced premature shutdown since they were expected to exceed the PTS regulatory limit before the end of the license period.

\section{RPV Simulation Model}

To demonstrate an initial proof of concept for Grizzly, the finite element model shown in Figure 1 was developed of a typical PWR reactor pressure vessel. 

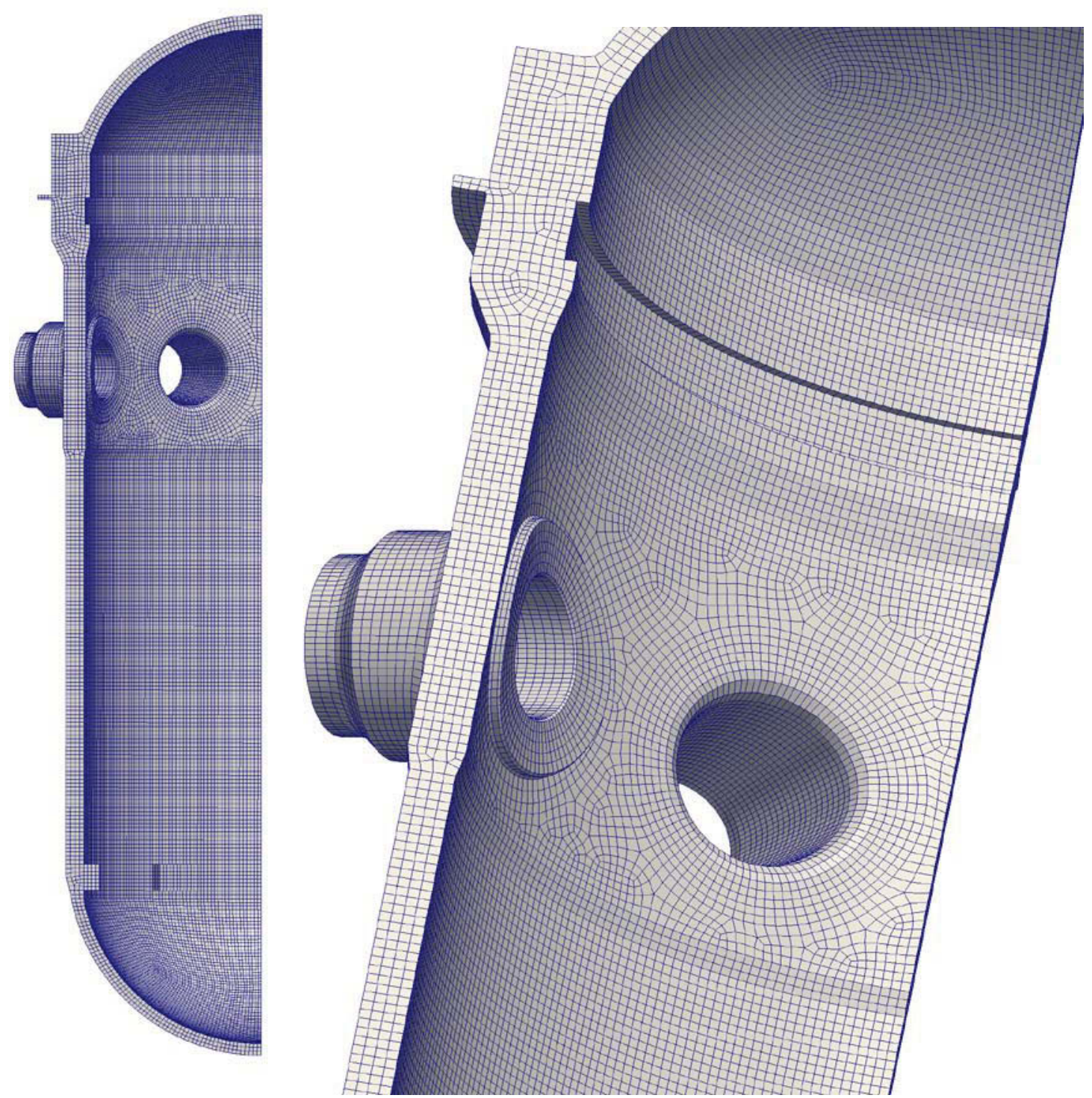

Figure 1: Overall and Detailed View of Finite Element Mesh

\section{Simplified RPV PTS Simulation Conditions}

The model shown in Figure 1 was subjected to the internal pressure history shown in Figure 2 and the coolant temperature history shown in Figure 3, using a time-varying heat transfer coefficient calculated by the RELAP 5 code. 


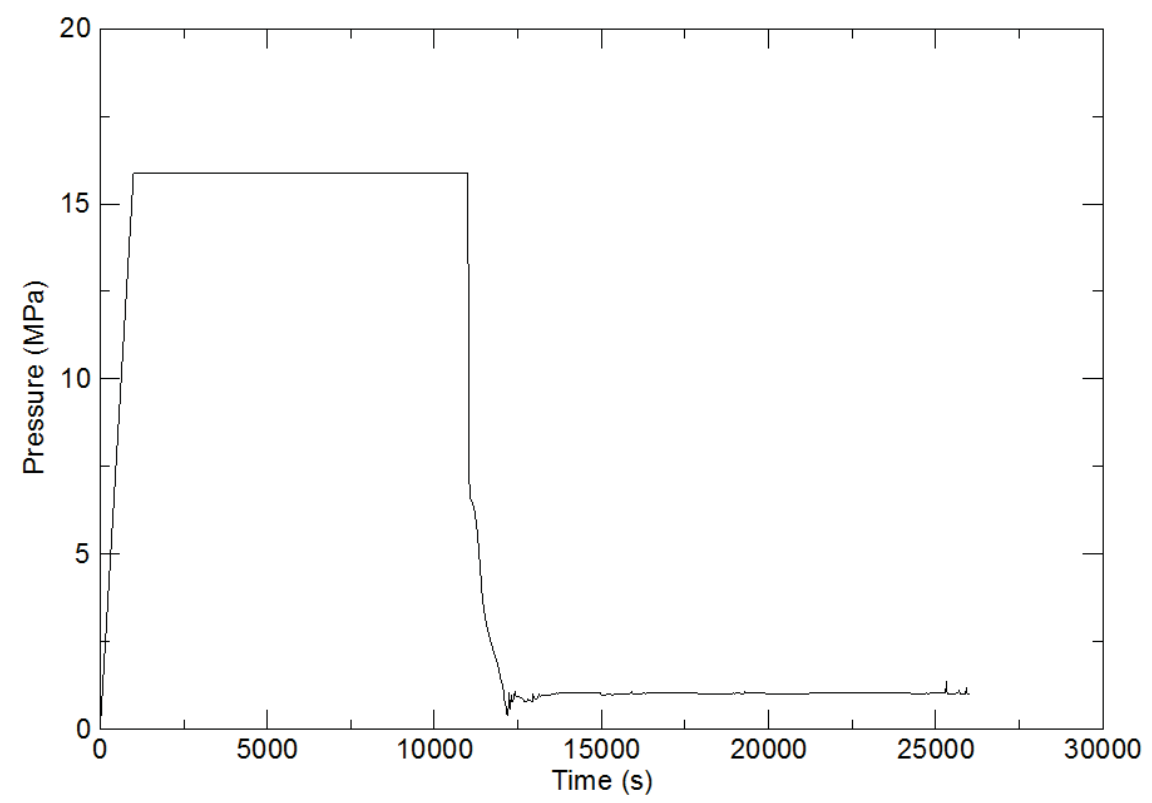

Figure 2: Pressure History

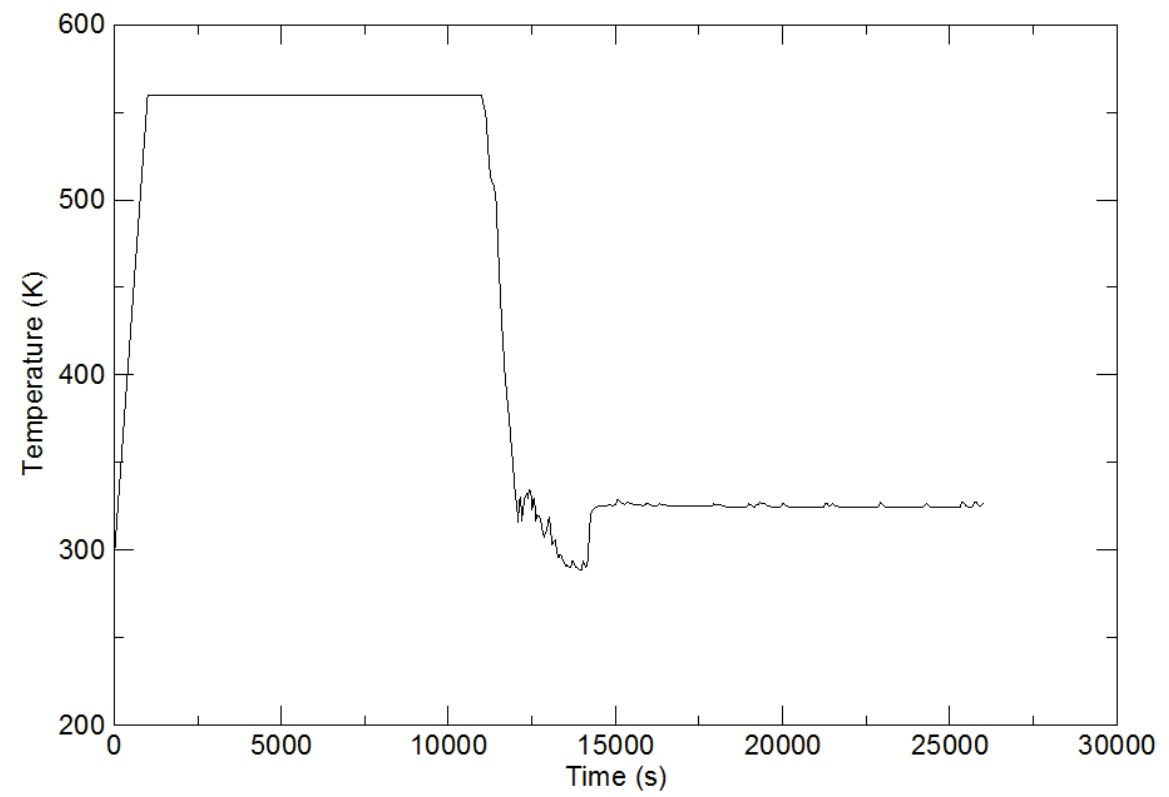

Figure 3: Coolant Temperature History 


\section{RPV PTS Simulation Results}

Figure 4 shows a typical fluence map for 32 effective full power years that was applied to the interior of the RPV beltline and attenuated through the wall thickness. The EONY model was used to calculate the shift in the transition temperature, also shown in Figure 4 , for every integration point in the model.
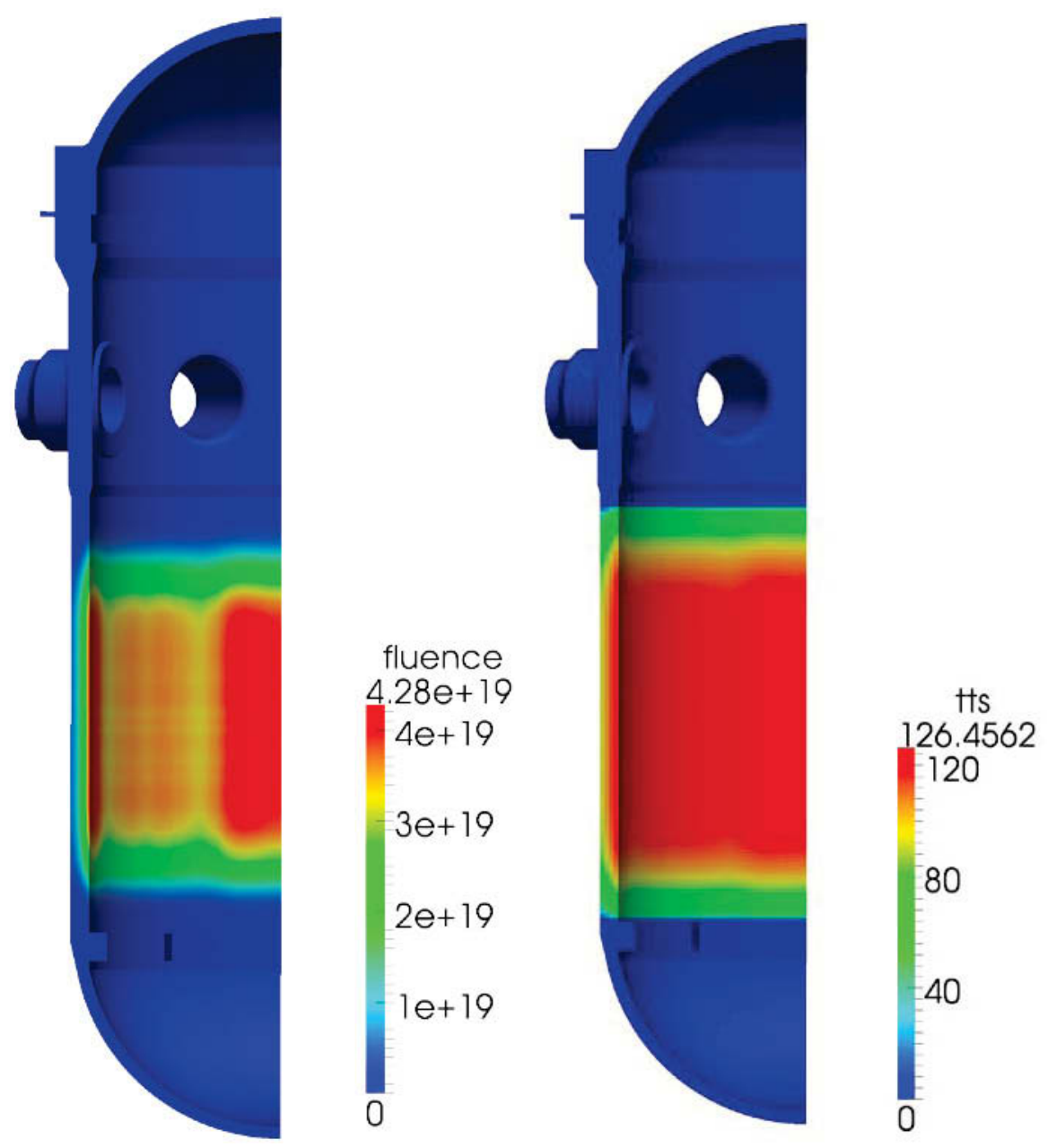

Figure 4: Fluence $\left(\mathrm{n} / \mathrm{cm}^{2}\right)$ and Reference Temperature Shift (Degrees F)

Figures 5 and 6 show the hoop stress and temperature contours at various points in time over the history of the transient event. These are shown at the beginning of the transient, midway through the pressure drop, at the point with the lowest pressure, immediately after the temperature rise, and at the end of the event. 

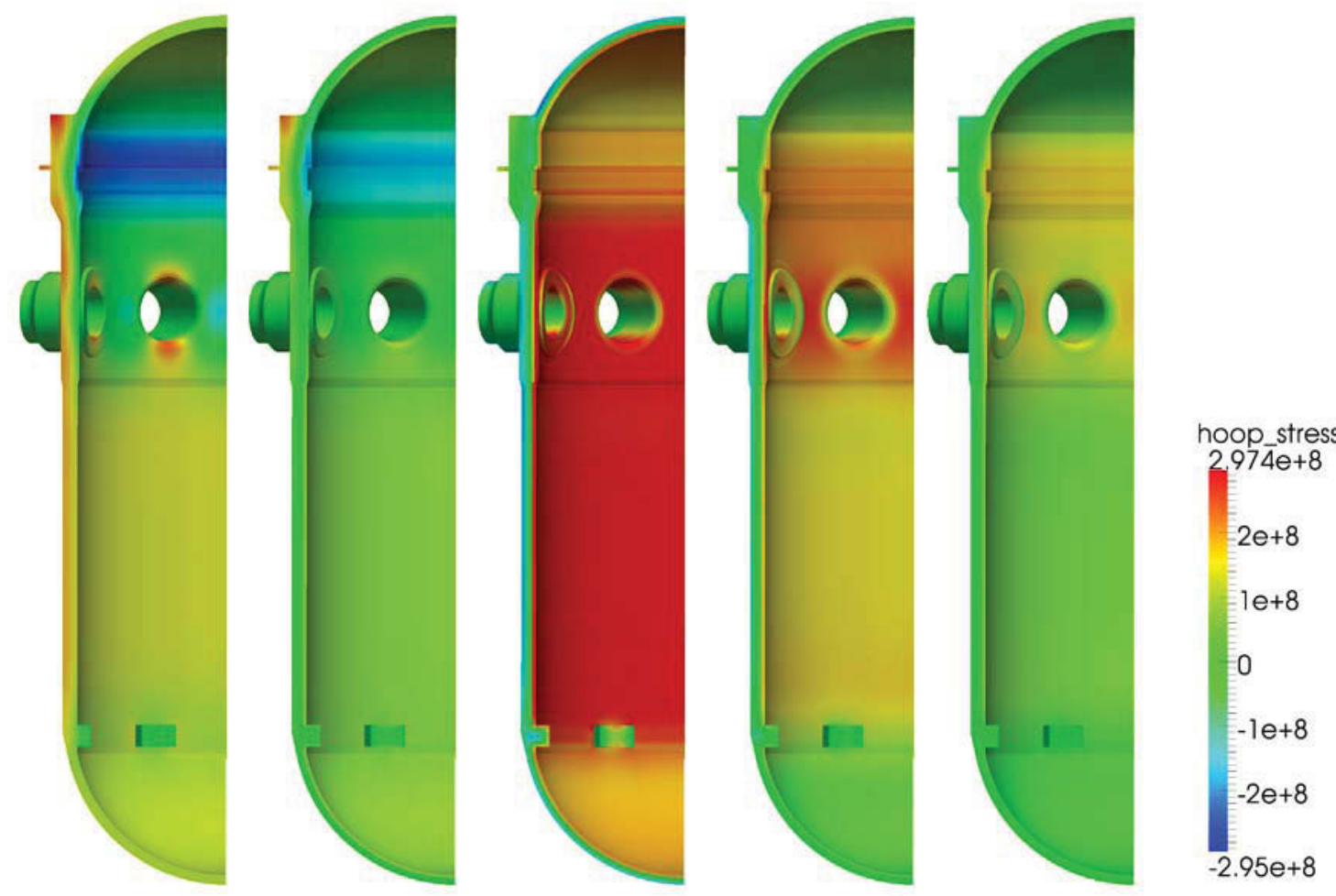

Figure 5: Hoop Stress (MPA) at points during re-flooding event


Figure 6: Temperature (K) During Re-Flooding Event 